\title{
24. ADICIONES AL CATÁLOGO DE LAS LABIADAS DE LA PROVINCIA DE MÁLAGA (España).
}

\author{
Baltasar CABEZUDO, José María NIETO CALDERA
}

Palabras clave: Lamiaceae, corología, Málaga, Andalucía, España.

Se comenta la presencia de dos especies de labiadas no citadas para la provincia de Málaga en un trabajo anterior (CABEZUDO et al. 1991).

Rosmarinus tomentosus L., Sp. Pl.: 23 (1753).

Especie considerada endémica del litoral granadino (ROSUA, 1986). Ha sido localizada en la provincia de Málaga en roquedos próximos al mar y de naturaleza calizo dolomítica. Incluida tradicionalmente en comunidades de Asplenietea (Rosmarinetum tomentosi Fedz. Casas \& López Guadalupe in Fdez. Casas 1972) la poblacion malagueña forma parte de un matorral incluible en la serie del RhamnoMayteneto que contacta con comunidades de Crithmo-Limonietea Br.-Bl. 1947.

Material estudiado: Málaga. Nerja, Cerro de la Caleta, Puntilla de Málaga (MGC 34248, 34424).

\section{Prasium majus L.}

Aunque frecuente en la provincia de Granada, no había sido citado para la provincia de Málaga. Habita en roquedos calizo dolomíticos, en la serie del RhamnoMayteneto (Lavandulo dentatae-Genistetum retamoidis Rivas-Goday y Rivas-Martínez 1968). Tambien puede aparecer en comunidades de derrubios y base de paredes verticales.

Material estudiado: Málaga. Nerja, Arroyo de la Miel (MGC 34287).

\section{BIBLIOGRAFÍA}

ROSÚA, J. L. -1986- Contribución al estudio del género Rosmarinus L. en el Mediterráneo occidental. Lagascalia, 14 (2): 179-187.

CABEZUDO, B., J.M. NIETO CALDERA y T. NAVARRO -1991- Catálogo de las labiadas (Labiatae) malacitanas (Málaga; España). Acta Bot. Malacitana, 16(2): 347-372.

(Aceptado para su publicación en Mayo de 1992)

Dirección de los autores. Departamento de Biología Vegetal. Facultad de Ciencias. Universidad de Málaga. Ap. 59. 29080 Málaga. 